\title{
“SI VOLVEMOS A VERNOS, TIERRA QUERIDA. EL PAPEL DEL ENTORNO NATURAL EN UNA MINA LLAMADA INFIERNO Y ZIA SUMMER"
}

\author{
(C) (1) (8) \\ María Antonia MezQuita Fernández ${ }^{1}$ \\ Universidad de Valladolid, GIECO-Franklin
}

\begin{abstract}
Resumen
Son muchos los críticos y autores que han manifestado un enorme interés por el tema de la justicia medioambiental. El siguiente artículo se centrará, a través de la disciplina de la Ecocrítica, en el estudio de dos novelas: Una mina llamada Infierno, del español Alejandro Gallo, y Zia Summer, del mexicano Rudolfo Anaya. A pesar de estar englobadas en el género de la ficción criminal, ambas novelas contienen una clara defensa del medio ambiente, por eso será interesante analizarlas.
\end{abstract}

Palabras clave

Justicia medioambiental, Ecococrítica, mina, Alejandro Gallo, Rudolfo Anaya.

\begin{abstract}
So many literary critics and writers have shown an abiding interest in environmental justice and the defence of the environment. This paper will show, through an ecocritical approaching, a comparative study on two novels: Una mina llamada Infierno, by the Spanish Alejandro Gallo, and Zia Summer, by the Mexican Rudolfo Anaya. In spite of belonging to crime fiction, both novels contain a clear defence of the environment. So, it will be interesting to analyze them.
\end{abstract}

Key words

Environmental justice, ecocriticism, mine, Alejandro Gallo, Rudolfo Anaya.

La Revolución Industrial trajo consigo una completa y compleja transformación, tanto del paisaje como de las condiciones de vida. Ante el inminente avance de la industria, las ciudades se llenaron de fábricas y máquinas que relegaron a un segundo plano el trabajo llevado a cabo en el campo. En el siglo XIX, algunas de las consecuencias del rápido crecimiento de la urbe fueron el empobrecimiento de la población, el hambre y el hacinamiento. En medio de esta situación social, la colectividad comenzó a imperar sobre la individualidad y la crítica se hizo eco de la parte más negativa del avance: la pérdida de identidad individual. Precisamente fueron los autores de ese periodo quienes se dieron cuenta de la importancia de enfatizar la subjetividad:

La Revolución Industrial condujo a una creciente reglamentación del individuo. Las pequeñas ciudades y pueblos, donde cada uno conocía a sus vecinos, iniciaron sociedad mecanizada, alimentada por la producción en masa. En este nuevo mundo, los individuos perdieron su identidad. Los escritores de esta época quisieron enmendar este desequilibrio dando importancia a la sensibilidad y a la conciencia individual. (Carter y McRae, 2004:

1 Universidad de Valladolid. Correo: mezquita@fing.uva.es. Recibido: 30-06-2014. Aceptado: 1-12-2014 
$202)^{2}$.

La literatura ha mostrado en todo momento un enorme interés por este tema, tanto por las consecuencias derivadas de la industrialización como por el efecto en el entorno natural y en la población. Así pues, son muchas las obras que se han centrado en esta situación en su evolución y en sus efectos. Pero no solo la literatura se ha ocupado de ello, dado que la crítica literaria también ha dirigido su mirada hacia las consecuencias producidas por el fenómeno de la industrialización. De entre las corrientes que han analizado dichas consecuencias, es notable destacar la Ecocrítica, que, en su preocupación por el medio ambiente, ha encontrado en la justicia medioambiental una vía para canalizar y denunciar las irregularidades y los efectos del deterioro, el abuso y la explotación del entorno natural.

El siguiente estudio se centrará en el análisis del medio ambiente en dos novelas donde la industria juega un papel primordial: Una mina llamada Infierno, de Alejandro Gallo, y Zia Summer, de Rudolfo Anaya. A pesar de tratarse de obras pertenecientes al género de la ficción criminal, de sus páginas se desprenden una fuerte crítica y una clara denuncia como resultado de la industrialización y de las consecuencias más negativas de ella. Dicha industrialización queda materializada en una mina, en el caso de Gallo, y en una planta de residuos nucleares, en el caso de Anaya. Veremos, por tanto, cómo el entorno condiciona la vida de los habitantes de la zona, su trabajo y los sentimientos que se producen en circunstancias laborales duras o en medio de una amenaza de contaminación o incluso de una posible catástrofe nuclear. Al final de las dos obras, lo que prevalece es la defensa del entorno y de sus gentes.

En base a todo lo anteriormente expuesto, es necesario comenzar dando una definición de lo que se entiende por ficción criminal y por justicia medioambiental, ya que nos servirá para enmarcar los acontecimientos que tienen lugar en ambas novelas y para entender algunos de los sentimientos que se dan en los personajes principales de las mismas. El género de la ficción criminal se caracteriza por tener como motivo central la resolución de uno varios crímenes con el propósito de restablecer el orden social. Por tanto, el fin de la ficción criminal es "investigar precisamente las "penumbras del alma", darnos no una "falsa", sino una verdadera psicología, penetrar en los dramas humanos y, a través de estos dramas, descubrir realmente unas y otras condiciones esenciales de la compleja realidad social (...)" (Galán Herrera, 2008: 59). En las dos novelas que vamos a analizar, el lector será testigo de lo que se oculta tras los asesinatos: especulación, drogas o destrucción del entorno natural, donde el drama

2 The Industrial Revolution led to an increasing regimentation of the individual. Small towns and villages, where everyone knew their neighbours, began mechanised society, fed and clothed by mass production. In this new world individuals lost their identity. The writers of this time wanted to correct this imbalance by giving greater value to the individual sensibility and to the individual consciousness. Todas las traducciones que se incluyen de fuentes secundarias son propias. 
humano tiene mucho más alcance de lo que parece a simple vista. Todorov plantea que en la novela negra coexisten dos historias: la del crimen y la de la investigación (en Scaggs, 2005: 3) ${ }^{3}$ y es justamente en medio de la segunda cuando sale a la luz un entramado que en ocasiones puede ser tan doloroso y complejo de resolver como el propio crimen en sí.

En lo referente a la justicia medioambiental, Laurence Buell, en The Future of Environmental Criticism. Environmental Crisis and Literary Imagination, afirma que los orígenes de la justicia medioambiental pueden rastrearse en Estados Unidos alrededor de 1980. El movimiento surgió como reacción a la contaminación medioambiental, los vertederos tóxicos y la industria. Normalmente, las comunidades más desfavorecidas han sido siempre las más perjudicadas (Buell, 2005: 141-2)4. En esta misma línea, en su artículo "Literatura, crítica y justicia medioambiental", Carmen Flys Junquera explica lo siguiente acerca de este movimiento:

La justicia medioambiental es uno de los movimientos activistas con mayor empuje en estos momentos. Dorceta Taylor afirma que el $68 \%$ de todos los movimientos ecologistas desde la década de 1980 pertenecen a esta corriente (...). Hay muchos y muy diversos movimientos de este tipo, pues se compone de pequeños grupos que forman redes en torno a un tema. Precisamente, una de las mayores dificultades se encuentra en su definición.

Taylor identifica cuatro corrientes del activismo medioambiental norteamericano en su artículo "Race, Class, Gender and American Enviromentalism" (2002). (Flys Junquera, 2020: 87).

De las cuatro corrientes que da Taylor, nos interesan la tercera y la cuarta por estar estrechamente relacionadas con el hilo conductor de las obras que vamos a analizar aquí. La primera tiene como objeto la contemplación y conservación de la naturaleza y la segunda se centra en el medio ambiente urbano. La tercera, en los derechos de los trabajadores (Fly Junquera, 2010: 87-89). Acerca de ella hay que apuntar que “[y]a desde mediados del siglo XIX, principalmente con la industrialización, empieza el activismo que promueve la seguridad y la salud laboral, la salud pública y los alojamientos decentes" (Flys Junquera, 2010: 98). En lo que se refiere a la cuarta corriente, se nos indica lo siguiente:

La cuarta corriente (...) es la conocida precisamente como la «agenda de justicia medioambiental». Esta tendencia se basa en los principios de justicia social y fue liderada por personas de «color» que se solidarizaron con la tercera corriente de la clase obrera. (...) Ambas tendencias se apoyan en el paradigma de la injusticia o la distribución de desigual de riesgos (...). Según Taylor, ya a lo largo del siglo XIX nos encontramos con grupos de protesta de los nativos americanos y méxico-americanos, que protestaron por la

3 In Todorov's analysis, the crime story contains two stories: the first is the story of the crime, and the second is the story of the investigation.

4 [E]nvironmental Justice is a rapidly growing grassroots movement, which started in the US around 1980 and a series of community-based resistance against toxification of local environments and the sitting of waste dumps and polluting industry that discriminate against poor and otherwise disempowered communities, particularly minority seen as victimized. 
expropiación de sus tierras y por las condiciones laborales en las minas (...). (Flys Junquera, 2020: 90).

Uno de los propósitos de la justicia medioambiental es dar voz a quienes denuncian las irregularidades cometidas en determinadas comunidades y tierras oprimidas por aquéllos que se consideran superiores. En su estudio American Indian Literature, Environmental Justice and Ecocriticism, Joni Adamson expone que en octubre de 1991 tuvo lugar la Primera Cumbre Nacional de Líderes Medioambientales de Color ${ }^{5}$ en Washington, donde se pudieron debatir y compartir experiencias en lo que se refiere al racismo medioambiental". El objetivo principal de la cumbre era "ampliar y profundizar en un proyecto político" que se basase en la justicia medioambiental y que asegurase a determinadas comunidades una liberación política, económica y cultural que durante 500 años de colonización había sido negada (Adamson, 2001: xvii) ${ }^{7}$. En la mencionada cumbre se materializaron los "Principios de Justicia Medioambiental", cuyas primera y última cláusula son las que mostramos a continuación:

La justicia medioambiental afirma el carácter sagrado de la Madre Naturaleza, la unidad ecológica y la interdependencia de todas las especies y el derecho a evitar una destrucción ecológica.

La justicia medioambiental requiere que nosotros, como individuos, elijamos de manera personal, consumir los mínimos recursos de la Madre Naturaleza y producir la menor basura posible. Y que, de forma deliberada, optemos por modelos de vida que aseguren unas condiciones óptimas del mundo natural para generaciones presentes y futuras. (Buell, 2005: 114$)^{8}$.

Con respecto a lo anterior y como ejemplo de comunidades más oprimidas de Estados Unidos, que es donde más estudios y debates acerca de este tema se han generado, destacan los afro-americanos, los nativos americanos, los latinos, los mexicanos y los asiáticos. En general, la relación que estas comunidades tienen con la tierra que habitan no es solo de dependencia sino también afectiva. Por razones históricas y culturales, en numerosas ocasiones han sido alienadas, siendo este el detonante de manifestaciones pidiendo igualdad y respeto para estas comunidades y sus tierras. Sin embargo, a juicio de Joni Adamson y pasando de lo universal a lo particular, son los propios

5 First National People of Color Environmental Leadership Summit.

6 Environmental racism.

7 At the Environmental Summit, delegates took a stand against environmental racism, drawing up Seventeen Principles of Environmental Justice that profiled a broad and deep political project to pursue environmental justice and secure a "political, economic and cultural liberation that has been denied for over 500 years of colonization and oppression (...)".

8 Environmental justice affirms the sacredness of Mother Earth, ecological unity and the interdependence of all species, and the right to bee free from ecological destruction.

Environmental justice requires that we, as individuals, make personal and consumer choices to consume as little of Mother's Earth resources and to produce as little waste as possible; and make the conscious decision to challenge and reprioritize our life-styles to insure the health of the natural world for present and future generations. 
grupos quienes también deberían hacer un esfuerzo y luchar por su supervivencia y por una mejora en las condiciones sociales, de vida y ambientales:

\begin{abstract}
Moviéndonos de lo universal a lo particular, esos grupos deberían trabajar por "la justicia", "salvar a la Madre Tierra", "cambiar las cosas de manera correcta" y "proteger la santidad de la naturaleza" como conceptos útiles y básicos para ayudarlos a hablar acerca de las necesidades que regulen las relaciones con los humanos y no humanos. Aunque, al mismo tiempo, su propósito será trabajar de forma colectiva por una serie de objetivos sociales y ecológicos en un lugar concreto. Moviéndonos en otra dirección, de lo particular a lo universal, ganar poder político les requerirá salir de sus asuntos particulares y aceptar una agenda de cambio social más universal. (Adamson, 2001: 74) ${ }^{9}$.
\end{abstract}

En el caso de España, las consecuencias de la Guerra Civil dejan al país sumido en una profunda crisis económica y social de la que tardaría años en recuperarse. A lo largo de la década de los 50, la crisis que se había venido experimentando en el campo se acentúa aún más. No obstante, los efectos de la industrialización comienzan a notarse cuando se produce paulatinamente un trasvase de inversiones del campo a la urbe. Aumenta, de este modo, el consumo y se generan mejores en la producción, aunque todo ello no está exento de huelgas y protestas. El éxodo del campo a la ciudad hace que se concentre un mayor número de población en las ciudades o en lugares eminentemente industriales donde la mano de obra barata será la que prime y la que tienda a contratarse. Una de las industrias que más se potencia en determinadas zonas del norte del país es la de la minería y especialmente la del carbón. Pero el crecimiento de esta industria no está exento de problemas:

La etapa autárquica supuso un nuevo período de crecimiento de la producción nacional de carbón. Ante la escasez de otras fuentes energéticas y las dificultades casi insalvables, al menos durante los años cuarenta, para adquirir recursos energéticos en el exterior, los planteamientos económicos de este período (...) no dejaban otra alternativa, para atender las necesidades derivadas de la reconstrucción y la industrialización, que fomentar la producción de carbón. Una producción que, al finalizar la contienda civil, resultaba claramente insuficiente para satisfacer la demanda. Ante esta situación y la práctica suspensión de las importaciones (...), garantizar el abastecimiento y evitar que la escasez impulsara alzas desmedidas en los precios se convirtieron en los principales objetivos de la política carbonera, en tanto que el problema de la falta de competitividad del carbón nacional, tan acuciante en los años anteriores a la Guerra Civil, era ahora irrelevante. (Sánchez Melado, 2007: 247).

Como ya hemos apuntado, en la década de los 50 se potencian las inversiones en la industria. Concretamente en la provincia de León, la minería aumentó considerablemente, tal y como puede observarse en la siguiente cita:

\footnotetext{
9 Moving from universality to particularity, these groups might employ certain notions of "justice", "saving Mother Earth", "changing things in a good way", or "protecting the sanctity of nature" as useful foundational concepts to help them talk about the need to regulate human relationships to other humans and nonhuman others, but at the same time, their goal will be to work collectively towards a particular set of ecological goals in a specific place. Moving in the other direction, from particularity to universality, they may find that gaining political power will require them to expand out from the confines of their particular issues of origin and embrace a more universal social change agenda.
} 
Los años cuarenta y cincuenta fueron decisivos para la industria nacional del carbón. Durante esas dos décadas surgieron o se desarrollaron muchas de las compañías que se iban a convertir en protagonistas de su devenir. En la provincia de León, Minero Siderúrgica de Ponferrada aumentó su producción un 75 por 100; Antracitas de Gaiztarro, un 69 por 100; la Vasco-Leonesa, un 298 por 100; Hulleras de Sabero, un 22 por 100; Antracitas de Fabero, que no se encontraba entre los productores de carbón en 1940, veinte años después era la tercera empresa carbonera leonesa por su volumen de actividad. (Sánchez Melado, 2007: 245).

Así “[e]n 1960 la plantilla carbonera leonesa, integrada por 21.825 trabajadores, duplicaba a la de 1940, año en el que se cifraron en 10.727 los puestos de trabajo generados por esta industria" (Sánchez Melado, 2007: 254). Por tanto, al aumentar la producción, era necesario aumentar la mano de obra. Pese a todo, se observa que el aumento de producción no implicaba que éste fuese directamente proporcional a la mejora de las condiciones laborales, pues como han evidenciado algunas investigaciones en el tema, la escasez de medios y la precariedad seguían siendo la tónica dominante:

Esta expansión del negocio carbonero, de la mano, en general, de empresas de notable dimensión no bastó para contrarrestar la escasez de medios y la precariedad del trabajo en las minas, cuya más trágica consecuencia la constituyeron los accidentes y las catástrofes que todavía hoy, por la frecuencia con la que se sucedieron, sobrecogen el ánimo de quien repasa la prensa de la época. Acontecimientos desgraciados que en las minas leonesas acabaron con la vida de 701 trabajadores entre 1940 y 1959, prácticamente un fallecido cada diez días. (Sánchez Melado, 2007: 260).

$\mathrm{Y}$, sin duda alguna, uno de los factores más importantes en el auge y el crecimiento de la industria minera fue la construcción del ferrocarril, lo cual facilitó el transporte entre zonas. Diversos estudios que se han realizado al respecto muestran la relevancia de este hecho y ponen de manifiesto el auge de la minería leonesa:

Por lo que en la provincia de León no es hasta finales del siglo XIX cuando la industria carbonera consigue despegar con la construcción del ferrocarril de La Robla (León) a Valmaseda (Vizcaya), inaugurado en 1894 y prolongado en 1901 hasta Luchana, ferrocarril que venía a solucionar el problemas de transporte del carbón leonés al posibilitarle llegar al País Vasco. Con la construcción del ferrocarril de La Robla se produce una explosión del interés hacia las cuencas carboníferas leonesas. (Casado Galán, 2009).

En las dos novelas que van a ser estudiadas, el entorno natural tiene un papel primordial, tanto en el desarrollo de los acontecimientos como en los sentimientos de afecto a esa tierra que se generan en los personajes principales. Alejandro Gallo centra la trama de Una mina llamada Infierno (2005) en la cuenca minera leonesa. Se trata de una comarca que conoce de primera mano debido a sus orígenes, puesto que nació en Astorga. Por tanto, el vínculo que le une con la minería tiene mucho que ver con su tierra natal y más tarde también tendrá que ver con su lugar de trabajo. El propio Gallo lo ha reconocido en varias ocasiones y ha querido enfatizar a la vez su vinculación emocional con la zona: 
Yo crecí en un pueblo minero, en el que la mina lo era todo para su gente. Después de un accidente grave en la mina, que casi le cuesta la vida a mi padre, vino una larga odisea por esos mundos: Alemania, Madrid, Toledo, León... Y en el año 97 recabé mucha información, como jefe de la policía local de Langreo. Langreo es el centro por antonomasia de la cuenca minera del Nalón, en Asturias, destino en el que estuve hasta el 2001, cuando me incorporé como jefe de la policía local de Gijón. Puedo decir, sin equivocarme, ni exagerar, que mi vinculación es visceral. (Gómez Cabezas, 2009).

El escenario que Gallo utiliza para enmarcar la trama tiene consecuencias fácilmente visibles en el desarrollo de los acontecimientos y en la manera de pensar y de actuar de los personajes principales de la novela. En Una mina llamada Infierno, la acción se desdobla en dos focos principales: Madrid y la cuenca minera leonesa. Madrid es el centro de operaciones y la base donde el protagonista, el inspector Ramallo da Costa, conocido como Trini, ha tenido siempre su lugar de trabajo y donde debe volver, una vez asentado en la comarca leonesa, cuando las circunstancias lo requieran. Una vez es enviado para resolver una serie de asesinatos a Vega del Bierzo, nombre ficticio que Gallo inventa pero que el lector puede reconocer fácilmente como un pueblo que podría localizarse en la comarca del Bierzo, Trini cambia sus costumbres y su manera de pensar. $Y$ todo ello viene condicionado por el modo de vida y el trabajo en este pueblo minero. La cuadrilla de Picas pasará, a partir de este momento, a formar parte de su vida cotidiana y se convertirá en algo más que un simple grupo de compañeros.

Una mina llamada Infierno refleja a la perfección el trabajo de los obreros en la mina y las dificultades que tienen a diario para llevarlo a cabo. De hecho, quizás muchos de los problemas a los que se han tenido que enfrentar siempre los trabajadores de la cuenca minera de León son los mismos a los que se enfrentan los personajes de la novela de Gallo. Al describir el terrible estado de la mina, donde no faltan humedades, goteras y condiciones insalubres y peligrosas, se hace una crítica de las pésimas condiciones laborales que tienen los operarios de Infierno:

\footnotetext{
Nos fuimos dirigiendo a una enorme nave, que debía de ser la de los vestuarios. Entré en ellos. Le puedo asegurar que nunca más volveré a protestar por los vestuarios de la comisaría, donde todo son quejas "que si no funciona la calefacción, que si los de la limpieza no pasaron hoy, que si alguien me tocó mi taquilla. (...)" Imagínese una nave de casi cincuenta metros de larga, cubierta de uralita, con alguna gotera que nadie se preocupa de reparar, las paredes decoradas con grandes manchas de agua $\mathrm{y}$, colgados de cuerdas que penden del techo, monos de mahón secándose, enganchados a cascos y lámparas. Cada uno se dirigió a su taquilla, menos yo, que desconocía cuál era mi destino. (...) Sólo se oía el ruido de las puertas metálicas de las taquillas que se abrían de golpe y el chirriar de los bancos de madera al ser arrastrados por aquel suelo de terrazo ennegrecido. (Gallo, 2005: 102).
}

Ante tal situación, podemos traer a colación las corrientes de activismo medioambiental que menciona Taylor y, concretamente, la tercera, que es la que se centra en la seguridad laboral y las condiciones de los trabajadores. En el caso de esta novela, las condiciones laborales que se muestran no son precisamente óptimas. Incluso el nombre que el autor da a la mina deja entrever la problemática existente y puede 
considerarse un preludio de los crímenes que tendrán lugar en ella. Probablemente, y de acuerdo con lo que hemos venido apuntando, Gallo conocía de primera mano las condiciones de algunas minas y ha optado por hacer una denuncia en primera persona, por medio de Trini. La forma en la que Gallo describe los personajes y los escenarios confieren una enorme credibilidad al texto, resultando totalmente verosímil. Acerca de este proceso creativo, él tiene su propia teoría:

Pero lo que siempre va a condicionar es el escenario en el que se muevan o en el que el autor los sitúe: no se mueve igual un protagonista por las noches de New York, que por las mañanas en un pueblo de Castilla, ni su lenguaje es el mismo si visita el Barrio de Salamanca o pulula por el cinturón industrial de Bilbao o las cuencas mineras. Si yo sitúo el escenario en las cuencas mineras de Asturias y León, debo crear un personaje creíble para los habitantes de allí, que se sumerja en su población como uno más. ¿Se imagina un personaje sibarita, algo dandy, bronceado por una máquina de rayos uva, picando carbón a quinientos metros bajo tierra con un martillo compresor de diez kilos en una mano durante diez horas? Si hablo de mineros, de guerrilleros antifranquistas, de huelguistas que caen abatidos por cuatro balas en la barriga, de gente que quisieron o quieren cambiar lo que les rodea, debo dibujarlos como son: recios, complejos, fieles a sus ideas y compañeros, éticos... (Gómez Cabezas, 2009).

En este mismo orden de ideas, un recurso propio de la ficción criminal es la crítica social. En la novela de Gallo, se critican las condiciones de los mineros que podrían estar inspirados, como ya hemos apuntado, en alguno de aquellos a los que Gallo conoció en la comarca donde creció o durante su estancia en Langreo. Mineros que no siempre realizaban su trabajo en las mejores circunstancias y de ahí que muchas veces sucediesen accidentes con consecuencias graves. Incluso la elección a la hora de contratarlos viene ya condicionada por el hecho de preferir a inmigrantes que no diesen problemas ni causasen revuelo: "Hace años que no meten a ningún español, sólo les interesan inmigrantes. Ya sabes, les pagan menos, protestan poco y se les puede mandar a los sitios más peligrosos sin que nadie diga una palabra" (Gallo, 2005: 67). Aunque los trabajadores no son los únicos que sufren las consecuencias de las malas condiciones laborales, pues la tierra también resulta afectada cuando se explotan los recursos naturales. Críticos como Joni Adamson hablan de "quienes se benefician al explotar los recursos naturales y explotar económicamente a los más débiles en guerras injustas o minas no seguras" (Adamson, 2001: 68) ${ }^{10}$ y diversos expertos en economía han alertado del peligro que supone la explotación de esos recursos:

La economía humana es un subsistema de un sistema físico más amplio. La economía recibe recursos (y a menudo los explota más allá de su capacidad de regeneración) y produce residuos. No existe una economía circular cerrada. Los perjudicados no sólo son otras especies no-humanas y las próximas generaciones de humanos (que no pueden protestar) sino que a menudo son también gente pobre, que protesta. (Martínez Alier, 2008:12).

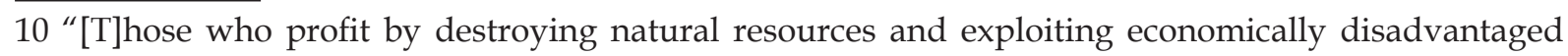
people in unjust wars o unsafe mines. 
En situaciones de desigualdad y de condiciones desfavorables como las que se mencionan anteriormente y las que se denuncian en la novela, a los trabajadores solamente les queda el derecho a la protesta para conseguir una mejora en su situación laboral. Protesta que no siempre se lleva a cabo en un primer momento, optando por la conformidad. Gallo describe a la perfección el suceso en boca del profesor Llago en los siguientes términos:

Después de la huelga, la cuenca quedó tranquila, no se movía nadie, el ritmo era habitual: por las mañanas y a primera hora de la tarde se veía la masa de obreros con monos azules dirigirse hacia el tajo, algunos salían de sus casas incluso con los cascos puestos, pocos se cambiaban en los vestuarios de las empresas. Por las noches, las tascas estaban llenas pero hasta horas prudenciales, pues al día siguiente había que ir a trabajar. Sólo se estaba hasta altas horas los sábados y domingos. Fue un ritmo de vida que no se rompió ni siquiera cuando decretaron el estado de excepción en todo el país a finales del 69. (Gallo, 2005: 121).

Pero con el paso del tiempo y tras ver cómo tienen que desarrollar su tarea diaria, los mineros de la cuadrilla de Picas, apoyándose en otros compañeros, se rebelan y deciden tomarse la justicia por su mano con un único propósito: presionar a la patronal para lograr una mejora notable en sus condiciones laborales.

\begin{abstract}
El objetivo estaba claro: aislar la cuenca al paso de la Guardia Civil. Hasta que restablecieran los puentes iban a pasar unos días, tiempo suficiente para que se pudieran hacer asambleas en los pozos y provocar una huelga general para presionar a la patronal, por los salarios, por la seguridad, por el trabajo los domingos. La gran huelga estaba preparada. (...)

La paralización del trabajo en las minas, las asambleas en las bocas de los pozos se dieron por todos los lados. (...) Pararon todos los pozos, menos los de la Minero SA. Sin embargo, a las doce del mediodía, una explosión dio al traste con el tendido eléctrico que suministraba a toda la empresa y no les quedó más remedio que detener el trabajo. (Gallo, 2005: 117-118).
\end{abstract}

Es característica propia del hard-boiled fiction el hecho de que el escenario del crimen esté enmarcado en la urbe. Sin embargo, en la novela de Gallo, la urbe será únicamente el lugar al que Trini se dirigirá para realizar parte de su investigación y donde surgirán historias paralelas que mostrarán la parte más oscura de la sociedad. En consonancia con lo que apuntaba Todorov, la investigación de los crímenes destapa los males más dañinos de la sociedad a los que el detective tiene que enfrentarse si quiere resolver el enigma y restaurar el orden. A pesar de pertenecer al género de la ficción criminal, uno de los propósitos más claros de esta obra es mostrar cuáles han sido las condiciones laborales en el entorno minero y, en consecuencia, hacer una crítica a dichas condiciones. Asimismo, otro de los temas que son objeto de denuncia por parte del autor es el de cómo la especulación afecta al entorno, a los trabajadores e incluso a la propia industria minera. Puede que el entramado que describe Gallo no diste mucho de lo que él mismo habrá conocido y experimentado de cerca durante su estancia en la comarca minera o incluso por su propia experiencia profesional:

Llevo mucho tiempo en este asunto de los delitos económicos y tengo una teoría. Estos chiringuitos financieros, como nosotros los llamamos, nacen cuando se producen dos 
fenómenos. El primero, una situación de crisis para un grupo numeroso de personas, en el caso que nos ocupa, la minería: se cierran los pozos, no hay puestos de trabajo para nadie, las prejubilaciones de la minería privada, que en realidad es colocar en el paro con cuarenta y tantos años a una gente a la que se le engaña con un incentivo de unos millones que no son suficientes para comenzar una nueva vida... Y puede ser cualquier otro colectivo. El segundo, un sector económico pujante con grandes beneficios que sea necesario ocultar para eludir impuestos, en este momento, la construcción. Así, parte de este dinero negro del sector necesita ser blanqueado y una forma de hacerlo es a través de esas financieras. (Gallo, 2005: 47).

A pesar de la evidente crítica social que subyace, Gallo muestra claras evidencias de cómo los trabajadores sienten un enorme apego por esa tierra y no desean abandonarla en ningún momento, defendiéndola de manera ferviente, pues ella es parte de su vida. Con el tiempo, Trini irá descubriendo que los asesinatos de la cuadrilla ocultan razones más oscuras y los trabajadores de la mina, que profesan un enorme afecto a su tierra, no son más que un estorbo al que hay que eliminar lo antes posible para conseguir el objetivo fijado:

Aquel hilo del que estaba tirando tenía una madeja muy significativa: los Vallona. Dueños del valle durante más de un siglo, inversores mayoritarios en la Financiera Berciana, especuladores natos que no dudaban en pagar a sicarios para conseguir sus objetivos $y$, en medio de todo eso, La Castañeda, en la cual estaba claro que pretendían hacer negocios y no dejarse pisar el terreno por los grandes grupos inmobiliarios. Pero les había nacido un grano en medio del trasero que no les dejaba sentarse: la mina situada en medio de esa gran extensión de tierra recientemente recalificada y que tenía unos inquilinos muy molestos. Los Vallona necesitaban expulsar a los arrendatarios y cerrar la mina para que ese terreno fuese también recalificado en una segunda fase. Los arrendatarios no eran otros que los miembros de la cuadrilla y, si todos iban muriendo, no habría arrendatarios molestos ni que hubiese que indemnizar. (Gallo, 2005: 148).

Es cierto que, en la actualidad, la preocupación por la ecología y la protección del medio ambiente ha aumentado considerablemente y esto puede observarse perfectamente en la literatura y en la crítica literaria, pero también en otros campos. En su libro The Voice of the Earth. An Exploration of Ecopsychology, Theodore Roszak muestra cómo el prefijo eco está empezando a unirse a otras áreas y disciplinas, lo cual es un indicativo del creciente interés que está surgiendo en torno al medio ambiente. Aunque para Roszak, quizás esta preocupación debería haber surgido mucho antes:

En estos días, vemos el prefijo "eco" añadido a muchas palabras. Ecopolítica, ecofilosofía, ecofeminismo, ecoconsumismo o, incluso, ecoterrorismo ... (...) [E]l gesto es relevante como un signo de los tiempos que vivimos. Esta pequeña bandera que tiene que ver con el uso de vocablos, planea sobre nuestra lengua como una tormenta amenazante intentando avisar de nuestra preocupación tardía por el destino de nuestro planeta. Su, a menudo, rara conexión con las palabras de muchos campos -política, economía, artes- revela el entendimiento creciente de cuántos aspectos de la vida que son preocupantes tendremos que aceptar. (Roszak, 2001: 14) ${ }^{11}$.

11 These days we see the prefix "eco" affixed to many words. Ecopolitics, ecophilosophy, ecofeminism, ecoconsumerism, even ecoterrorism. ... The result is not always graceful, but the gesture is nonetheless significant as a sign of the times. This tiny neologistic flag flies above our language like a storm-warning 
Por otra parte, críticos como Simon C. Stock consideran que hasta que no entendamos la forma en la que estamos actuando tan negativamente con el mundo natural y nos aseguremos de que se respetan sus derechos, será imposible que nuestras acciones sobre el mismo tengan un efecto positivo, dado que:

A menos de que estemos hablando de la preocupación en aumento por entender cómo nuestros pensamientos y nuestras acciones infringen los derechos del mundo natural, además del intento manifiesto de garantizar los derechos legales del medio ambiente (...), es, sin duda, complicado que influyamos cómo utilizamos el mundo natural que tenemos actualmente -lo cual es prácticamente nulo-. (Stock, 2011: 31) 12. $^{2}$

Enlazando directamente con el tema de los derechos medioambientales, la siguiente novela que analizaremos será la del escritor chicano Rudolfo Anaya, Zia Summer (1995), que, además de estar englobada en el género de la ficción criminal y al igual que en la novela de Gallo, aboga claramente por asegurar el bienestar y la protección del medio ambiente. Uno de los aspectos más interesantes de las novelas de Anaya, y especialmente en el caso de la que vamos a estudiar aquí, es el interés que muestra por el entorno natural y por las creencias y supersticiones de su gente. Anaya recupera antiguas creencias y ritos de su pueblo y las mezcla con historias creíbles, dando lugar a un tipo de novela englobada dentro de lo que se ha calificado como "Realismo mágico" que, de acuerdo con Imelda Martín Junquera, experimenta "un retorno a un modelo colonial, a una función adánica de nombrar las cosas" (Martín Junquera, 2005: 13). Estamos, por tanto, ante "un regreso al pasado o una recreación de un supuesto pasado tribal" (Martín Junquera, 2005: 13). En esta misma línea de conexión con el pasado, Martín Junquera apunta lo siguiente:

Para el escritor chicano se han invertido los términos de la oposición civilización naturaleza salvaje, pasando la primera a representar el caos del que los personajes se intentan alejar en busca de un mundo más en comunión con la naturaleza, muy en la tendencia rural de Faulkner. Quizá este hecho representa un retorno a las raíces, a un pasado primitivo en armonía con la naturaleza como postulan los nativo americanos, puesto que el chicano es también habitante indígena del continente americano. Esta relación de recuperación de las tradiciones y del folklore se produjo en un momento en el que la asimilación hacia la cultura angloamericana dominante fue tan notable que provocó un rechazo por parte de muchos chicanos de su lengua, religión y tradiciones. Entre los autores que mejor representan esta situación en sus obras, destaca Rudolfo Anaya (...). (Martín Junquera, 2005: 56).

Y si Alejandro Gallo se centró en una tierra que él conocía bien, gracias a sus vivencias,

meant to signal our belated concern for the fate of the planet. Its often awkward connection with words from many sources -politics, economics, the arts- reveals our growing realization of how many aspects of our life that concern will have to embrace.

12 Unless we are talking about extending/raising awareness with the explicit aim of understanding how both our thoughts and our actions infringe upon the rights of the natural world with the overt intent of moving towards ensuring legal rights for the environment in the same way that legal rights have been hard won for people, it is doubtful indeed that we will have very much more effect on how we use the natural world than we currently have -which is virtually nil. 
en Zia Summer Anaya nos transporta a Alburquerque, Nuevo México, que también conoce a la perfección por el hecho de haber nacido en Pastura. Por consiguiente, el papel que juega el entorno en esta obra es importante, dado que la vuelta a la naturaleza y a las raíces propias es un indicativo de que el entorno natural marcará el carácter de los personajes y los hechos que acontecen. Como sucedía con Alejandro Gallo, las novelas de Anaya no están exentas de crítica y de denuncia medioambiental, ya que:

La saga de Sony Baca de Anaya, con cuatro novelas, (...) muestr[a] las relaciones complejas entre los problemas del medio ambiente, las injusticias históricas, las desigualdades sociales y la globalización. Anaya introduce creencias de los Indios Pueblo en la re-escritura de la conquista de Nuevo México. Sony aprende a venerar la naturaleza y a conocer el potencial de sus sueños, a la vez que trabaja casos de tráfico de armas, drogas, experimentación con animales y la progresiva desertización de la zona a raíz de la construcción de una presa. (Flys Junquera, 2010: 117).

A diferencia de Trini, inspector de policía, Sony Baca, protagonista de la novela que vamos a tratar, es un detective privado que investiga el asesinato de su prima Gloria; asesinato que está envuelto en un halo de misterio por la mezcla de superstición y elementos tribales. La naturaleza tiene un papel sumamente relevante en este suceso, dado que es en ella donde se celebran todos los ritos y ceremonias ancestrales que se describen en la obra. El valle de Río Grande se diferencia claramente de la ciudad $\mathrm{y}$, en sus encuentros con los habitantes de dicho valle, Sony descubre, al igual que descubrió Trini, que sus sentimientos y su apego a este lugar se van acrecentando paulatinamente. Baca no es bien recibido al principio, pues mezclarse en la naturaleza implica, además de resolver el crimen, descubrir otros secretos turbios que no interesa que salgan a la luz porque acarrearían problemas. Por ello, lo que escucha por boca de quienes no lo ven con buenos ojos es: "We know you came to our mountain to cause trouble. This is a sacred place and we don't you here. Leave!" (Anaya, 1995: 137) ${ }^{13}$.

Por otra parte, la representación de la naturaleza como algo sagrado y la serie de ritos ancestrales confieren a la novela un encanto especial, aunque ese encanto se consigue también, como sucedía en el caso de Una mina llamada Infierno, debido a la visualidad y la credibilidad con la que el autor describe, de nuevo en primera persona omnisciente y por boca del protagonista, el entorno, las ceremonias o los pensamientos que se aglutinan en la mente su mente:

A clean, sulky smell filled the bright air. Thin clouds moved in from the southwest, high thin clouds that carried no rain but were the harbingers of the monsoon season. Cattle stayed close to the windmill water tanks. Overhead, two large turkey vultures circled. The constant wind swept mournfully across the dry grass. The land was quite and empty.

Sony squinted into the distance and wondered about the women who had survived the land and weather. First, the Indian women, the nomadic Comanches, then the Mexican women of the Quarai and Abo missions, finally the gaunt-facies Okies. So many either went crazy or died dreaming of water. Water was the element of survival. Without water there was no life on the burning land. Life gathered around the oasis of the windmill tanks.

13 Puesto que la novela no ha sido traducida al español, mantendremos la versión original en las citas. 
Each new tribe added its bits of technology to the land and thus changed the landscape. Human life could exist only within the radius of the small oasis that men created. (Anaya, 1995: 199).

De la cita anterior se desprende que la tecnología y el progreso se mezclan con la naturaleza. En Zia Summer, se observa cómo el avance tecnológico va imponiéndose a la belleza de los elementos naturales. Anaya considera que la naturaleza está comenzando a ser destruida por la mano del hombre, quien también necesita del progreso para sobrevivir y por eso es capaz de plasmar en su obra algo que se nos antoja tan contradictorio como que "la vida se junta en torno a ese pequeño oasis de los tanques de molinos de viento" (Anaya, 1995: 199). No obstante, la novela rezuma una dura crítica al progreso que destruye el valle y que no beneficia a las comunidades más desfavorecidas, en este caso, los nativos americanos. Este hecho podría relacionarse directamente con la cuarta corriente de justicia medioambiental de Taylor, ya que promueve la igualdad y la mejora en las condiciones laborales y con la "agenda de justicia medioambiental", puesto que trata de defender a personas de color y comunidades más oprimidas. A menudo, son estas comunidades las que tienen que sufrir efectos fatídicos en el medio ambiente, como por ejemplo la contaminación o la desertización. A juicio de Adriana Espinosa, todo ello suele repercutir en dichas comunidades, dado que:

(...) la falta de equidad en el acceso a los recursos naturales y en la carga de contaminación, en perjuicio de las poblaciones más vulnerables (ya sea por su origen étnico, su nivel de ingresos, su posición en la economía mundial, etc.) En otras palabras, son situaciones de conflictos ecológicos distributivos, que constituyen el objeto de estudio de un campo interdisciplinar de reciente creación, la Ecología Política. (Espinosa González, 2012: 53).

En ambas novelas, los autores realizan una férrea crítica a determinados males sociales como la especulación urbanística e inmobiliaria y a la destrucción del entorno natural. En el caso de Zia Summer, algunos de los habitantes del valle son plenamente conscientes de su situación y manifiestan su disconformidad: "“If you move a blade of grass, you change the land. If you poison the water, someday you will have to drink it," don Eliseo said. "But the government don't listen when the poor complain. The government don't listen to los pobres"'" (Anaya, 1995: 123). Evidentemente, en boca de don Eliseo, uno de los ancianos del valle, Anaya hace una crítica voraz al hecho de que "los pobres" no sean tenidos en cuenta y la contaminación de sus aguas o sus tierras les afecte más directamente que a otros sectores de la población. Y es que, si Gallo criticaba las condiciones de los trabajadores de la mina, sometidos a largas y duras jornadas y sin apenas seguridad en el pozo, Anaya hace lo mismo con los habitantes del valle, pero, en este caso, lo que denuncia es la contaminación, los residuos nucleares, la radioactividad y las graves consecuencias que todo ello puede acarrear: 
That high-level waste already kills people! Did you ever think of that? Tons and tons of it all over the world. Don't tell me what kills people, Baca! The feds, DOE, Sandia Labs, Los Alamos. They kill people! They killed your father. They've been storing high-level shit in this state for forty years. Sandia Labs has dumped right into the South Valley. Wise up, Baca. We didn't create the problem, we're trying to solve it. The DOE and the Defense Department have stockpiled nukes in the Manzano Mountains. All that stuff they're storing and dismantling is seeping into the water! At WIPP the barrels will be corroded by the salt! It's poisoning the earth! (...) (Anaya, 1995: 209).

En cualquier caso, Zia Summer podría ser considerada una novela de "terrorismo medioambiental" o, como indicaba Roszak, ecoterrorismo, dado que el tema de la contaminación y los residuos nucleares va más allá de la mera aparición. Ya en las primeras páginas de la novela se nos habla de la presencia de grupos anti WIPP, que más tarde tendrán un papel clave en el desarrollo de la trama y en la resolución del caso: "The anti-WIPP groups were threatening action if the Departmento of Energy went ahead with the proposed Waste Isolation Pilot Plant test" (Anaya, 1995: 7-8). Una vez más, Anaya ha trasladado su historia a un escenario real. Situado cerca de Carlsbad, Nuevo México, WIPP (Waste Isolation Pilot Plant) es "el primer servicio geológico diseñado para la eliminación de residuos radiactivos generados a causa de las actividades de defensa de los Estados Unidos" (United States Environmental Protection Agency, 2002) $)^{14}$. Los residuos son empaquetados y colocados dentro de cilindros metálicos para ser luego llevados hasta la planta, por tratarse de material contaminado con plutonio, americio y otros elementos radioactivos: "transporting material laced with plutonium from Los Alamos Labs down to the WIPP site near Carlsbad." (Anaya, 1995: 8).

El hilo conductor de la novela, el asesinato de Gloria, hace que el lector sea testigo de un intento de explotar uno de esos camiones cargados con cilindros metálicos que están siendo transportados a la planta de residuos. Si en la novela de Gallo asistíamos al relato de la voladura de un puente para evitar el transporte y el suministro y, de este modo, aislar la mina, aquí vemos un intento de provocar un desastre nuclear que pondría en riesgo a todo el valle y su población. Evidentemente, las razones en ambos casos no son las mismas y las consecuencias del segundo serían fatídicas, sobre todo para los habitantes de la zona. El propio autor no duda en emplear el término "terrorista medioambiental": "Blowing up a truck full of nuclear waste? Sony wondered. What the hell did he mean? Did the FBI think Raven was an environmental terrorist?" (Anaya, 1995: 143). El desastre se llega a evitar, pero Anaya aprovecha la ocasión para dejar patente que es la comunidad chicana que vive en el valle de Río grande a la que se ha perjudicado con la construcción de la planta:

But the deadly waste was here, and it was here to stay. It's life would outlive generation of the Río Grande valley. His children would live with the consequences. Somewhat it had to

14 The WIPP is the nation's first geological facility designed for permanent disposal of transuranic (TRU) radioactive waste that was generated as a result of the U.S. defense activities. 
stop. Somewhere men and women had to come to their senses and stop producing what they could not control. (Anaya, 1995: 308).

Sin embargo, y como ya sucedía con los negocios e intereses ocultos que había detrás de los asesinatos en Una mina llamada Infierno, Zia Summer esconde todo un entramado de intereses ocultos, especulación, negocios oscuros e inversiones extrañas con el único propósito de obtener beneficios de la comunidad que vive en el valle y de explotar sus recursos para sacar un alto rendimiento económico:

That's why Dominic is so damn dangerous, Sony thought. He wants to use the water of the Río Grande for his outrageous development, create a Disneyland for tourists, but he doesn't understand the balance of the river and the underground water play in the scheme of things of the Río Grande basin. He wants to build an oasis out of Alburquerque. Canals, green beltways, flower gardens, the flow of fountains, canals boats carrying passengers from one casino to the next. (Anaya, 1995: 199)

A medida que Sony avanza en su investigación, ve que va surgiendo una maraña de negocios oscuros, intereses ocultos y un extraño mercadeo que le conducen a pensar que el asesinato de su prima esconde razones más poderosas de lo que él creía en un primer momento. La similitud con la novela de Gallo está clara, pues en ella los miembros de la cuadrilla de Picas iban siendo asesinados con el fin de no impedir que los planes que quienes estaban llevando a cabo especulaciones urbanísticas llegasen a buen fin. Zia Summer tampoco contiene únicamente la historia de un asesinato, dado que los negocios turbios que se desarrollan alrededor del mismo son una parte muy importante de la trama:

Dominic had enough pull to have a special session of the state legislature convened in May, but much to everyone's surprise, it voted against the casino bill for the city. Conservative Republicans joined the Little Texas cowboy legislators from the southeast corner of the state in torpedoing the gambling bill. Suddenly the worm had turned on Dominic's bandwagon. Lawsuits against Dominic's corporation, which was buying Río Grande water rights, began to hit the courts, and Dominic's grand plan of building a Venice on the Río Grande ground to a standstill. (Anaya, 1995: 244).

Básicamente, y de acuerdo con lo que hemos podido observar, Alejandro Gallo y Rudolfo Anaya describen una tierra a la que sienten suya por ser parte de sus vivencias y por generar en ellos sentimientos de apego. Posiblemente, estos lugares estarán siempre en su memoria y serán, de forma involuntaria, una referencia continua en sus obras. Citando a David Sobel, "para los adultos, lugares especiales de su infancia son puntos de referencia de la memoria o lugares a los que vuelven continuamente con su mente" (en Thomashow, 1996: 10) ${ }^{15}$. El afecto que le profesan se percibe en dos de los fragmentos que aparecen en las últimas páginas de las novelas, aunque el tono de ambas cambia bastante. En las palabras de Gallo, se advierte una veta de tristeza por

15 [F]or adults, special places from childhood serve as "touchstone memories," or places to which they continually return in their mind's eye. 
la situación de la industria minera y de los trabajadores. El deterioro del paisaje y de la salud de los mineros pone la nota melancólica en la novela y deja entrever el hecho de que no solo se está destruyendo el entorno natural, sino también el bienestar de las gentes de la zona:

\begin{abstract}
Estos parajes encierran la energía legendaria de luchas libradas de las que emergieron héroes y sueños que no llegaron a ser. (...) Sus vidas se pierden por las edificaciones que se derrumban y en los hogares sometidos a una gran prueba. A cada paso que damos contemplamos y sentimos el dolor de la explotación y la piel se nos llena del polvo negruzco del carbón que se extiende por los caminos. Las aguas de sus ríos pierden la cristalina transparencia y sus peces se metamorfosean para sobrevivir. $Y$ hasta la amargura que se respira es más peligrosa que la silicosis. (Gallo, 2005: 227).
\end{abstract}

La idea que se desprende de las de Anaya es que, al menos por esta vez, la tierra se ha salvado. El tono es más positivo que el de Gallo, ya que, aunque Anaya es consciente de que el peligro está ahí, la naturaleza podrá continuar su ciclo y la vida se regenerará, puesto que se ha podido evitar el desastre. No obstante, el peligro está latente y puede que se desencadene la tragedia en un futuro no muy lejano. Aunque ahora no es el momento y el mundo natural continuará su curso:

Tonight the earth will not be burned by the deadly heat of radiation (...) and tomorrow the sun would shine, and after the rain, the slaked earth of summer would green again.

Time and again, the seasons came and renew the Earth. The drought was broken and now the grasses of the land would green up. (Anaya, 1995: 307).

En suma, y conforme a lo que ya hemos apuntado con anterioridad, Una mina llamada Infierno y Zia Summer son dos novelas pertenecientes al género de la ficción criminal cuyo fin último es investigar una serie de asesinatos que han tenido lugar en medio del entorno natural. A medida que el investigador avanza, descubre un entramado de negocios oscuros cuyas consecuencias podrían ser nefastas para los habitantes de la zona y que se extenderían también al paisaje: la especulación urbanística o la destrucción del medio ambiente, junto con la explotación, las operaciones oscuras y el blanqueo de quienes pretenden aprovecharse y sacar beneficio de manera ilegal. Por tanto, la trama de ambas obras es bastante más compleja de lo que a simple vista parece. Se podría decir que las dos tienen una estructura en forma de telaraña donde todo está conectado y es producto de una acción anterior.

Al mismo tiempo, la crítica social que realizan ambos autores queda patente a lo largo de ambas novelas. Si bien tal crítica no se limita únicamente a la social, pues tanto Alejandro Gallo como Rudolfo Anaya, a pesar de estar distanciados en espacio, son conscientes de cómo el mundo natural marca y conforma la vida los habitantes de un lugar y del daño que puede causar la destrucción o la contaminación del mismo. En el caso de Una mina llamada Infierno, Gallo hace mención a las enfermedades derivadas del mineral extraído y también a las pésimas condiciones de las galerías y de los pozos. En Zia Summer, Anaya nos pone en la hipótesis de lo que podría pasar ante un desastre 
ecológico de semejante magnitud como sería la voladura de los tanques con residuos radioactivos. Una mina llamada Infierno y Zia Summer contienen una parte de justicia medioambiental importante y una clara defensa del entorno natural como algo que no debe ser destruido y que tendrá que perdurar intacto. En ambas novelas, el entorno influye en el modus operandi del asesino, pero también en la forma de pensar y de actuar del personaje principal. Por consiguiente, el detective, se va acomodando y adaptando paulatinamente a las situaciones sobrevenidas. Trini y Sony se integran y se adentran en las costumbres de los habitantes de Vega del Bierzo y del valle de Río Grande respectivamente y, aunque el proceso de integración es más evidente y mayor en el primero, en el segundo se aprecia un interés emergente por las tradiciones ancestrales y por las creencias de su pueblo. Con el tiempo, llegará un momento en que los dos se convertirán ya en un habitante más de la zona y serán plenamente conscientes de que el lazo que los ha unido a ella no se romperá nunca.

En las dos novelas, el afecto que sienten Gallo y Anaya por el entorno natural se manifiesta de forma clara y se refleja y aprecia perfectamente en los protagonistas. Trini y Sony quedarán atados para siempre a esa tierra; tierra a la cual volverán y que se queda en su corazón. Los dos son sabedores del hecho de que hay que cuidarla, dado que solamente así ella nos cuidará a nosotros. Lo contrario nos conduciría a error, pues "[1]uchar contra la Madre Tierra significa destruir y corromper, matar, envenenar. Si hacemos eso, no tendremos paz. La primera paz llega con tu madre, la Madre tierra"16. (Chief Oren Lyons, 2013: 11).

\section{BibLIOGRAFÍA}

Adamson, J. (2001): American Indian Literature, Environmental Justice and Ecocriticism, Tucson, The University of Arizona Press.

Buell, L. (2005): The Future of Environmental Criticism. Environmental Crisis and Literary Imagination, Malden, Blackwell Publishing.

Carter, R. y McRae, J. (2004): The Routledge History of Literature in English. Britain and Ireland, Oxon, Routledge.

Espinosa González, A. (2012): “La justicia ambiental, hacia la igualdad en el disfrute del derecho a un ambiente sano", Universitas. Revista de Filosofía, Derecho y Política, $16,51-77$.

Flys Junquera, C. (2010): "Literatura, crítica y justicia medioambiental", en C. Flys Junquera et alii (eds.) (2010) Ecocríticas. Literatura y medio ambiente, Madrid, Iberoamericana / Vervuert: 85-119.

Galán Herrrera, J. J. (2008): “El Canon de la novela negra y policiaca”, Tejuelo, 1, 58-74.

Gallo, A. (2005): Una mina llamada Infierno, Oviedo, Editorial Laria.

Gómez Cabezas, J. R. (2009): “Entrevista con Alejandro Gallo", http://gangsterera. free.fr/entrev_AGallo.htm (Consultado en marzo de 2010).

16 To make war against Mother Earth is to destroy and to corrupt, to kill, to poison. When we do that, we will not have peace. The first peace comes with your mother, Mother Earth. 
Lyons, O. (2013): “Listening to Natural Laws”, en Vaughan-Lee, Ll. et alii (eds.) (2013) Spiritual Ecology. The Cry of the Earth, Point Reyes, The Golden Sufi Center.

Martínez Alier, J. (2008): “Conflictos ecológicos y justicia ambiental", Papeles, 13, 11-27.

Martín Junquera, I. (2005): Las literaturas chicana y nativo americana ante el realismo mágico, León, Secretariado de Publicaciones.

Roszak, T. (2001): The Voice of the Earth. An Exploration of Ecopsychology, Grand Rapids, Phanes Press, INC.

Sánchez Melado, J. (2007): “La minería leonesa del carbón durante la autarquía”, Estudios Humanísticos. Historia, 6, 245-271.

Scaggs, J. (2005): Crime Fiction, New York, Routledge.

Stock, S. (2011): "Ecocritical Theory, Presentism and Praxis", en S. Oppermann et alii (eds.) (2011) The Future of Ecocriticism. New Horizons, New Castle upon Tyne, Cambridge Scholars Publishing.

Thomashow, M. (1996): Ecological Identity. Becoming a Reflective Environmentalis, Cambridge, Massachusetts, The MIT Press.

United States Environmental Protection Agency (2002): "Regulating the Safety of the WIPP", http:// www.epa.gov/radiation/docs/wipp/wippsafety1.pdf(Consultado en junio de 2014). 